OPEN ACCESS

Edited by:

Mihajlo Jakovljevic,

University of Kragujevac, Serbia;

Hosei University Tokyo, Japan

Reviewed by:

Mohsen Shams,

Yasuj University of

Medical Sciences, Iran

Ana Maria Moreno Londoño,

Consultant in Health Communication,

Switzerland

*Correspondence:

Silvia Riva

silvia.riva1@unimi.it

Specialty section:

This article was submitted

to Health Economics,

a section of the journal

Frontiers in Public Health

Received: 23 April 2016

Accepted: 23 May 2016

Published: 13 June 2016

Citation:

Riva S and Pravettoni G (2016)

Value-Based Model: A New

Perspective in Medical

Decision-making.

Front. Public Health 4:118. doi: 10.3389/fpubh.2016.00118

\section{Value-Based Model: A New Perspective in Medical Decision-making}

\author{
Silvia Riva ${ }^{1,2 *}$ and Gabriella Pravettoni ${ }^{1,2}$ \\ ${ }^{1}$ Department of Oncology and Onco-Hematology, University of Milan, Milan, Italy, ${ }^{2}$ Applied Research Division for Cognitive \\ and Psychological Science, European Institute of Oncology (IEO), Milan, Italy
}

Keywords: value-based decision-making, medical decision-making, medical decisions, health-related quality of life, patient outcomes

The problem of how to use financial resources beneficially in health care, despite limited budgets, reduced personnel, and inadequate time, goes beyond the realm of health economists (1). How do we use existing resources to prioritize one health program over another? Does a specific program provide a real and measurable improvement in terms of perceived health-related quality of life (HRQOL)? What decisions should we make?

In the recent years, the HRQOL index has become an asset for evaluating patient health status in health programs, therapies, and clinical trials (2). Created as a specific psychological construct for use in health contexts, the HRQOL index is now widely used and evaluated by health economists, epidemiologists, clinicians, and nurses. Today, the notion that health status is not only determined by survival or absence of disease but also closely related to quality of health is universally accepted $(3,4)$.

Health-related quality of life has been defined as the functional effect of a medical condition and/or treatment on a patient. Thus, HRQOL is a subjective and multidimensional parameter that encompasses physical and occupational functioning, psychological state, social interaction, and somatic sensation (5). Health is perceived by the person as a state that lies beyond the presence of a disease or the severity of a condition. HRQOL is influenced by several factors that include not only psychological and emotional aspects, individual behavior, and attitudes but also personal experiences, culture, and religious beliefs $(6,7)$. Owing to this subjectivity, patients with the same medical condition may have very different perceptions of HRQOL. For example, patients with some hematological conditions, such as hemophilia, may experience a good HRQOL despite having several disease-related complications, whereas others with the same disorder but better clinical conditions may perceive a poor HRQOL (8-12). Similar results have been obtained in patients with other chronic conditions, such as back pain $(13,14)$.

Evaluation of HRQOL has been encouraged over the last two decades to obtain a broader perspective of health status in patients and to identify which therapeutic choices and programs to pursue (15). Health economists may use the HRQOL construct as a deciding factor in applying economic analyses. For example, in cost-benefit, cost-effectiveness, or cost-utility analyses, HRQOL may represent one indicator for measuring effectiveness (e.g., success of a health program, specific treatment, or regulatory policy) (16). In this context, effectiveness may be measured as a final outcome, such as the number of life-years gained or number of lives saved, by applying a certain program or proposing a certain treatment. Alternatively, it may be measured as an intermediate outcome in a health program, such as the number of days lost from school or work, perceived HRQOL, treatment satisfaction, or psycho-emotional well-being (17).

The HRQOL construct focuses on the patient's perceived state of health as a reference value in evaluating programs and making medical decisions. In this light, we wish to discuss the potential of the HRQOL and psychological science in a new medical paradigm that has been recently described as an alternative approach to the traditional paradigm of evidence-based medicine, namely, the value-based medicine approach. 


\section{PSYCHOLOGICAL PERSPECTIVE OF THE VALUE-BASED SYSTEM}

Health systems are increasingly being burdened by enormous public expenditures, and health policy decisions made to contain costs have so far been ineffective. A new direction for facilitating health-care management and containing health-care costs may be represented by Porter's theoretical value-based model $(18,19)$. According to this model, choices in the health context should be oriented toward "adding value for the patient." Health systems may support interventions and choices that appear expensive in the short term but provide better outcomes in the long term, with a drastic reduction of costs and public expenditures (20).

The value-based model is different from the evidence model, but they are linked together. While the traditional evidence-based medicine often measures the improvement gained in length of survivorship, but generally does not measure the significance in terms of quality-of-life improvement, the value-based medicine integrates the best results of evidence-based medicine (it uses best evidence-based data) to a superior level of analysis by combining the HRQOL evaluations of patients "with a disease in concerning the value of an intervention" (20).

According to this perspective, it is necessary to promote integrated interventions that care for the whole patient, with the psychological aspects of care taking on increasing importance $(21,22)$. The value-based model highlights the importance of health outcomes, including HRQOL and patient well-being, in making appropriate medical decisions. Sustainable and improved health-care management and costs may be obtained by measuring concrete patient health outcomes (patient utility) and abandoning evaluations of general health structure costs, which had been proposed by the traditional evidence-based medicine model (20). The value-based model proposes that health outcomes be measured over a longer period, and that the patient's health status be compared in a comprehensive and integrated manner that considers all aspects of the patient's life (i.e., physical health, psychological aspects, and social life). Thus, the term value cannot be reduced to the cost of an intervention but must include a general evaluation of the patient's overall well-being. Although this general evaluation includes HRQOL, the construct must be evaluated in a wider perspective together with all other health outcomes (e.g., utility, satisfaction, patient preferences, and level of functioning). According to Porter, health outcomes should be measured by medical condition (e.g., cancer), not by specialty (e.g., oncology) or intervention (e.g., chemotherapy). More importantly, health outcomes should be considered across the full period of care for the condition and should track the patient's health status after conclusion of care (23).

In practice, the consideration of health outcomes, including HRQOL, represents only one tier for the development of an

\section{REFERENCES}

1. Keehan SP, Cuckler GA, Sisko AM, Madison AJ, Smith SD, Stone DA, et al. National health expenditure projections, 2014-24: spending growth faster than recent trends. Health Aff (Millwood) (2015) 34:1407-14. doi:10.1377/ hlthaff.2015.0600 effective valued-based system in health care. Other tiers may include the development of (1) integrated health-care practice units comprising clinical and non-clinical health-care team members; (2) bundled payment systems that cover the full care cycle over a defined period of time or for a defined population, based on improvement of health outcomes; (3) integrated care delivery systems able to eliminate fragmentation of care; and (4) local network opportunities among clinics and medical institutions (24). All of these practical proposals may favor a change from the evidence-based model (centered purely on scientific evidence and organized around what physicians do) to a valued-based model (centered on the patient and organized around what patients need) $(23,24)$. The value-based medicine model seems to comply with the definition of health reported by the World Health Organization (WHO): "Health is a state of complete physical, mental, and social well-being and not merely the absence of disease or infirmity" (25).

Health outcomes measure the effects of treatment and care that patients receive. Outcomes include, for example, remission of the condition, degree of patient functionality, recovery status, and emotional well-being. The value-based medicine model highlights the importance of the psycho-social dimensions, which enrich the traditional clinical picture by describing the patient not only as sick but also a person with a specific biophysical and psychological profile who is inserted in a social context (family, work, etc.). The direction proposed by value-based medicine indicates that hospitals need to place the patient in the center, evaluating and integrating all human and social aspects of a person.

According to this model, outcome indicators need to be appropriate and specific for each particular condition. They should not be measured for a single event or over a short time frame but rather should be measured throughout the entire course of treatment (e.g., inpatient, outpatient, and day hospital phases). This perspective advocates the importance of a personalized approach to medicine, particularly the Five Ps of Personalization, which includes a Predictive, Precise, Preventive, Participatory Medicine that is sensitive to Psychological aspects and the patient's cognitive characteristics (26). In particular, consideration of the psychological aspects requires an investigation of the psycho-cognitive variables that come into play when each individual makes a personal choice (27-29) with respect to the health, treatment, and care pathways.

\section{AUTHOR CONTRIBUTIONS}

SR conceived the work, reviewed literature, and wrote the manuscript. GP supervised the work and wrote the manuscript. All the authors have agreed on the final version of the manuscript.

2. Krasuska M, Riva S, Fava L, von Mackensen S, Bullinger M. Linking qualityof-life measures using the international classification of functioning, disability and health and the international classification of functioning, disability and health-children and youth version in chronic health conditions: the example of young people with hemophilia. Am J Phys Med Rehabil (2012) 91(13):S74-83. doi:10.1097/PHM.0b013e31823d4f35 
3. Haas BK. A multidisciplinary concept analysis of quality of life. West J Nurs Res (1999) 21(6):728-42. doi:10.1177/01939459922044153

4. Vallerand AH, Payne JK. Theories and conceptual models to guide quality of life related research. 2nd ed. In: King CR, Hinds PS, Sudbury MA, editors. Quality of Life: From Nursing and Patient Perspectives. Sudbury, MA: Jones \& Bartlett Publishers (2003). p. 45-54.

5. Schipper H, Clinch JJ, Olweny CLM. Quality of life studies: definitions and conceptual issues. In: Spilker B, editor. Quality of Life and Pharmacoeconomics in Clinical Trials. Philadelphia, PA: Lippincott-Raven Publishers (1996). p. 11-23.

6. Bullinger M. Assessing health related quality of life in medicine: an overview over concepts, methods and applications in international research. Restor Neurol Neurosci (2002) 20:93-101.

7. Mueller PS, Plevak DJ, Rummans TA. Religious involvement, spirituality, and medicine: implications for clinical practice. Mayo Clin Proc (2001) 76(12):1225-35. doi:10.4065/76.12.1225

8. Riva S, Cutica I, Krampe C, Reinecke LF, Russell-Edu W, Santoro C, et al. A cohort pilot study on HIV-associated neuropsychological impairments in hemophilia patients. Front Hum Neurosci (2015) 9:313. doi:10.3389/ fnhum. 2015.00313

9. Riva S, Nobili A, Djade CD, Mancuso ME, Santagostino E, Pravettoni G. Cognitive and psychological profiles in treatment compliance: a study in an elderly population with hemophilia. Clin Interv Aging (2015) 10:1141-6. doi:10.2147/CIA.S84749

10. Mangiafico L, Perja M, Fusco F, Riva S, Mago D, Gringeri A. Safety and effectiveness of raltegravir in patients with haemophilia and anti-HIV multidrug resistance. Haemophilia (2011) 18(1):108-11. doi:10.1111/j.1365-2516.2011.02610.x

11. Riva S, Cutica I, Pravettoni G. Neuropsychological profile in a specific cohort of HIV patients infected postnatally: a cross-sectional study. Neurobehav HIV Med (2015) 6:1-6. doi:10.2147/NBHIV.S84838

12. Riva S, Cutica I, Pravettoni G. Is there evidence for neurocognitive dysfunctions in patients with postnatal HIV infection? A review on the cohort of hemophilia patients. Front Hum Neurosci (2014) 8:470. doi:10.3389/ fnhum.2014.00470

13. Riva S, Camerini AL, Allam A, Schulz PJ. Interactive sections of an internet-based intervention increase empowerment of chronic back pain patients: randomized controlled trial. J Med Internet Res (2014) 16(8):e18. doi:10.2196/ jmir.3474

14. Schulz PJ, Hartung U, Riva S. Causes, coping, and culture: a comparative survey study on representation of back pain in three Swiss language regions. PLoS One (2013) 8(11):e78029. doi:10.1371/journal.pone.0078029

15. Bullinger M, Quitmann J. Quality of life as patient-reported outcomes: principles of assessment. Dialogues Clin Neurosci (2014) 16:137-45.

16. Feeny DH, Eckstrom E, Whitlock EP, Perdue LA. A Primer for Systematic Reviewers on the Measurement of Functional Status and Health-Related Quality of Life in Older Adults (Internet). Rockville, MD: Agency for Healthcare Research and Quality (US); Patient-Reported Outcomes, Health-Related Quality of Life, and Function: An Overview of Measurement Properties (2013). Available from: http://www.ncbi.nlm.nih.gov/books/NBK169156/
17. Eandi M, Pradelli L, Zaniolo O. Principi e scopi della farmacoeconomia. Profili Farmacoeconomia (2006) 1. p. 5-8.

18. Porter ME. A strategy for health care reform - toward a value-based system. $N$ Engl J Med (2009) 361(2):109-12. doi:10.1056/NEJMp0904131

19. Porter ME. What is value in health care? N Engl J Med (2010) 363:2477-81. doi:10.1056/NEJMp1011024

20. Brown GC, Brown MM, Sharma S. Value-based medicine: evidence-based medicine and beyond. Ocul Immunol Inflamm (2003) 11(3):157-70. doi:10.1076/ocii.11.3.157.17355

21. Porter ME, Larsson S, Lee TH. Standardizing patient outcomes measurement. N Engl J Med (2016) 374(6):504-6. doi:10.1056/NEJMp1511701

22. Roe RH, Lass JH, Brown GC, Brown MM. The value-based medicine comparative effectiveness and cost-effectiveness of penetrating keratoplasty for keratoconus. Cornea (2008) 27(9):10017. doi:10.1097/ICO.0b013e $31817 \mathrm{bb} 062$

23. Porter ME, Teisberg EO. Redefining Health Care: Creating Value-Based Competition on Results. Boston, MA: Harvard Business School Press (2006).

24. Porter ME, Pabo EA, Lee TH. Redesigning primary care: a strategic vision to improve value by organizing around patients' needs. Health Aff (2013) 32(3):516-25. doi:10.1377/hlthaff.2012.0961

25. WHO Definition of Health. Preamble to the Constitution of the World Health Organization as Adopted by the International Health Conference, New York, 19-22 June, 1946; Signed on 22 July 1946 by the Representatives of 61 States (Official Records of the World Health Organization, no. 2, p. 100) and Entered into Force on 7 April 1948. Available at: http://www.who.int/about/definition/ en/print.html [accessed May 27, 2016].

26. Pravettoni G, Gorini A. A P5 cancer medicine approach: why personalized medicine cannot ignore psychology. J Eval Clin Pract (2011) 17(4):594-6. doi:10.1111/j.1365-2753.2011.01709.x

27. Lucchiari C, Pravettoni G. Cognitive balanced model: a conceptual scheme of diagnostic decision making. J Eval Clin Pract (2012) 18(1):82-8. doi:10.1111/j.1365-2753.2011.01771.x

28. Gorini A, Pravettoni G. An overview on cognitive aspects implicated in medical decisions. Eur J Intern Med (2011) 22(6):547-53. doi:10.1016/j. ejim.2011.06.008

29. Baldi PL, Iannello P, Riva S, Antonietti A. Cognitive reflection and socially biased decisions. Stud Psychol (2013) 55(4):265-71.

Conflict of Interest Statement: The authors declare that the research was conducted in the absence of any commercial or financial relationships that could be construed as a potential conflict of interest.

Copyright (๑) 2016 Riva and Pravettoni. This is an open-access article distributed under the terms of the Creative Commons Attribution License (CC BY). The use, distribution or reproduction in other forums is permitted, provided the original author(s) or licensor are credited and that the original publication in this journal is cited, in accordance with accepted academic practice. No use, distribution or reproduction is permitted which does not comply with these terms. 